
\title{
25 Research Soure \\ The Effect of Different Ventilation Modes on the Outcomes of Patients Infected with Coronavirus Disease 2019 (COVID-19): a Protocol of Systematic Review and Network Meta-Analysis
}

Yihao Zhu



Fei Wang

Department of Anesthesiology, West China Hospital of Sichuan University

Tao Zhu ( $\nabla$ xwtao_zhu@yahoo.com )

Department of Anesthesiology, West China Hospital of Sichuan University

\section{Protocol}

Keywords: Coronavirus Disease 2019, COVID-19, SARS-CoV-2, Ventilation Model, Network Meta-Analysis

Posted Date: November 17th, 2020

DOI: https://doi.org/10.21203/rs.3.rs-108442/v1

License: (9) This work is licensed under a Creative Commons Attribution 4.0 International License.

Read Full License 


\section{Abstract}

Background: Coronavirus disease 2019 (COVID-19) outbreak has spread around the world, the high mortality rate and strong infectious cause surging global patients diagnosed patients and death while in response to the outbreak, a myriad of scientific research workers and researchers have made unremitting efforts, but effective treatments are still limited, even may say that there is no specific treatment. So a large number of patients with severe patients need treatment for respiratory support, in particular, based on various realistic factors, different way of ventilation is widely used in clinical, which kind of ventilation way is the best one of the most effective ventilation strategy is not clear, so we planned a network metaanalysis to evaluate different ventilation methods on new crown the efficacy and safety of patients, expect to find an optimal ventilation strategy.

Methods and analysis: Two authors will independently search the electronic databases, preprints databases, Clinical Study Registration website and COVID-19 research related project database from December 1, 2019 to November 5, 2020. The primary outcomes are 1) All-cause mortality; 2) Transmission of COVID-19 to health care workers and other people; 3) Length of hospital stay; 4) Length of ICU stay. A systemic review and a network meta-analysis based on Bayesian framework will be performed to assess the effect of different ventilation modes on the outcomes of patients infected with COVID-19. The Grading of Recommendations Assessment, Development and Evaluation System (GRADE) will be used to evaluate the quality of evidence.

Discussion: COVID-19 has spread around the world and become a global public health security problem. With limited treatment available, a large number of critically ill patients need ventilator support treatment, and the demand for ventilators has increased sharply. To our knowledge, this study will be the first systematic review and NMA to analyze the efficacy and safety of different ventilation modes in patients with COVID-19. This study expected to obtain the best choice of ventilation mode for COVID-19 patients based on high quality evidence.

Ethics and dissemination: Ethical approval is not required owning to it is a literature-based study. The final conclusion will be disseminated through peer-reviewed publication.

\section{PROSPERO registration number: CRD42020219581}

\section{Background}

In early December 2019, a severe acute respiratory syndrome coronavirus virus 2 (SARS-CoV-2), named coronavirus disease 2019 (COVID-19), broke out in Wuhan, China, and has now spread globally. As of 5 November 2020, more than 48 million novel coronavirus infections had been diagnosed and over 1.22 million deaths had been confirmed worldwide ${ }^{[1]}$. In response to the outbreak, despite the tremendous efforts of scientists around the world and a total of 2,462 registered studies ${ }^{[2]}$, effective treatments are still limited and no specific treatment has been found. Therefore, a large number of patients need ventilator support treatment. A large number of studies are under way on the effects of different 
ventilation modes on COVID-19 patients. Recent studies on ventilation therapy have shown that early tracheotomy improves prognosis in patients with COVID-19 ${ }^{[3-5]}$, but some previous studies have shown that early tracheotomy does not improve mortality, but reduces the incidence of pulmonary complications ${ }^{[6,7]}$. In addition, as a result of the COVID-19 pandemic, there has been a surge in demand for ventilator equipment, we need to use all kinds of ventilators more rationally.

Therefore, we plan to conduct this network meta-analysis to acquire the best choice between non-invasive ventilation, tracheotomy and invasive ventilation in the early stage of COVID-19 patients, further analyze whether different ventilation parameter settings have an impact on patient outcomes, and find the optimal choice.

\section{Objective}

The purpose of this study is to investigate the effect of different modes of ventilation on outcome in COVID-19 patients through NMA and systematic review.

\section{Method}

The protocol of this review was first registered with the International Prospective Register of Systematic Reviews (PROSPERO), and Reporting Items for Systematic Reviews and Meta-Analysis Protocols (PRISMA-P) statement (Supplement S1) ${ }^{[8]}$ was followed while conducting and reporting the protocol of this systematic review and network meta-analysis (http://www.prisma-statement.org).

\section{Eligibility Criteria}

\section{Type of study}

The review will include randomized controlled trials (RCTs), case control study and cohort study that were reported in English or Chinese without any regional restrictions, irrespective of publication status and publication year. Case report, animal experiment, vitro cell experiment, expert experience, conference article, review, communication letter, comment and duplication publication will be excluded.

\section{Type Of Patients}

Patients with confirmed COVID-19 infection and hypoxemic respiratory failure (despite oxygen therapy) in any setting that has capacity for non-invasive ventilation (NIV) or invasive ventilation.

\section{Type Of Intervention}

In this systematic review, we defined NIV as continuous positive airway pressure (CPAP), biphasic positive airway pressure (BiPAP), proportionally assisted ventilation (PAV), and high-flow nasal catheter inhalation (HFNC).The invasive ventilation was tracheotomy, endotracheal intubation and laryngeal mask airway, while ventilator ventilation model was performed by pressure-controlled ventilation (PCV), volume- 
controlled ventilation (VCV), synchronous intermittent instruction ventilation (SIMV), spontaneous breathing support (PSV) and pressure-controlled volume-guaranteed ventilation (PCV-VG).

\section{Type Of Control Group}

Control treatments included early noninvasive or invasive ventilation, tracheotomy, sham operative ventilation. Studies comparing the therapeutic effects of different ventilation modes will also be included.

\section{Type Of Outcome Measures}

Studies that reported one or more of the following outcomes will be included.

\section{Primary outcome}

1. All-cause mortality.

2. Transmission of COVID-19 to health care workers and other people.

3. Length of hospital stay.

4. Length of ICU stay.

\section{Secondary outcome}

1. Complications of therapy.

2. Secondary bacterial pneumonia.

3. Need for invasive ventilation.

4. Need for tracheostomy.

5. (Time to) Recovery from COVID-19.

6. Aerosol generation and droplet dispersion of live virus at various distances and times.

7. Contextual outcomes including acceptability, feasibility, resources use and impact on equity.

8. Medical staff infected COVID-19.

\section{Type Of Language}

We will only include only English or Chinese literature.

\section{Search Method}

Two authors will independently search the electronic databases Web of Science, Embase, and the Cochrane Central Register of Controlled Trials (CENTRAL), Medline through Pubmed from December 1 , 2019 to November 9, 2020. We will search the Chinese electronic databases: Chinese National Knowledge Infrastructure (CNKI), Wanfang Database, VIP Database, Chinese Biomedical Literature Database (CBM), and we will also search World Health Organization (WHO) Clinical Trials Registry, Chinese clinical registry, ClinicalTrials.gov, ProQuest Central, SciFinder, the Virtual Health Library, LitCovid, WHO covid-19 website, 
Centers for Disease Control (CDC) covid-19 website, Eurosurveillance, China CDC Weekly. We will also search bioRxiv (preprints), medRxiv (preprints), chemRxiv (preprints), and SSRN (preprints), Chinese Medical Journal Net (preprints), and ChinaXiv (preprints).

A subject-specific search strategy (Supplement S2) was developed in Pubmed/Medline and applied as the basic search strategy in the other electronic databases. We also scanned the reference lists and citations of the included trials and any relevant systematic reviews, referring to further literature. If necessary, we contacted trial authors to obtain additional information.

\section{Data Collection}

Two investigators independently extracted the data of all included studies using a standard data exaction form prepared by authors, on which they recorded the first author, publication year, study design, ventilation modes, population characteristics, and reported outcomes of interest. If necessary, we contacted the authors of included studies by email to obtain additional data missing from the published paper. If additional information could not be obtained in this manner, we extracted data and information from the figures. At each step of data extraction, we resolved any differences between the investigators by discussion or consulting an arbiter.

\section{Study Selection}

Two authors independently scanned the title and abstract of the identified records to exclude uncorrelated studies and accessed the full-text articles for eligibility. We resolved any disagreements regarding inclusion of the study by discussion or by consulting an arbiter. The study selection procedure will be performed according to the PRSIMA flow chart (Figure. 1).

\section{Risk-of-bias In Individual Studies}

Two authors will respectively assess the risk-of-bias of all included studies, using the Cochrane risk-ofbias tool. We will appraise each study in terms of selection bias (random sequence generation and allocation concealment), performance bias (blinding of participants and personnel), detection bias (blinding of outcome assessment), attrition bias (incomplete outcome data), selective reporting bias and other bias. Each component was judged as low risk-of-bias (green), high risk-of-bias (red), or unclear riskof-bias (yellow).

\section{Quality Assessment}

The Grading of Recommendations Assessment, Development and Evaluation System (GRADE) will be used to evaluate the quality of the evidence for primary outcomes ${ }^{[9]}$. Two reviewers will independently assess the quality of evidence using GRADE software, the quality of evidence will be divided into four levels: high, medium, low and very low, according the GRADE standards. For a specific study, the quality will be assessed according to the risk of bias, inconsistency, indirectness, imprecision and publication bias. 


\section{Assessment Of Similarity And Consistency}

The assessment of similarity and consistency will be conducted to get a reliable and valid result. At present, it is difficult to determine similarity by statistical analysis, so we will evaluate the similarity of included studies by analyzing clinical and methodological characteristics, patient characteristics, experimental design, and interventions. We will use the z-test to check for consistency, and calculate the P-value to check for inconsistencies between direct and indirect comparisons and a P-value greater than $0.05(P>0.05)$ is considered to be consistent between the direct comparison and the indirect comparison, on the contrary, there is inconsistency.

\section{Network Meta-analysis}

Data synthesis and statistical analysis will be performed using R3.6.3 (https://www.r-project.org/) with the Bayesian framework in this NMA. For continuous outcomes, standard mean difference (SMD) with $95 \%$ confidence intervals (Cls) or a weighted mean difference (WMD) with $95 \%$ Cls will be applied to analyze the efficacy data, while risk ratio (RR) with $95 \%$ Cls will be used for dichotomous data. WMD will be used for the same units or for the same measurement method while SMD will be used for different scale or assessment tools.

The contribution of each study to the final effect will be assessed and demonstrated by mapping the netheat map. $P$ value will be used to measure the accuracy of different ventilation modes over the control group and different ventilation modes were ranked by $P$ value. $P$ value equal to 100 percent, it will be considered as the best treatment for patients, while $\mathrm{P}$ value equal to 0 percent, it will be considered the worst treatment. The results of the NMA will be shown using forest maps, the ranking results of different ventilation modes will be displayed by using the surface under the cumulative sorting curve analysis, and the network evidence plot will be applied to present the comparison between different interventions.

\section{Assessment Of Heterogeneity}

For the heterogeneity of clinical and methodological heterogeneity, the sources of heterogeneity will be assessed by careful assessment of participant characteristics, study design, randomization, blinding, interventions, and outcomes of all included studies. The $\mathrm{I}^{2}$ statistics was used to report statistical heterogeneity, which was classified by applying the Cochrane Handbook of Systematic Reviews of Interventions. When $\mathrm{I}^{2}>50 \%$, obvious heterogeneity existed, on the contrary, when $\mathrm{I}^{2}<50 \%$, heterogeneity is considered acceptable. Furthermore, if $\mathrm{I}^{2}>50 \%$, several subgroup analyses and sensitivity analyses were conducted to evaluate the source of heterogeneity and to assess the subpopulation of patients that could probably obtain benefits from the intervention. The final NMA will be performed after the removal of studies that produce primary or unacceptable sources of heterogeneity. Lastly, if the source of heterogeneity cannot be explored after subgroup analyses and sensitivity analyses, a narrative review will be provided.

\section{Meta-regression, Subgroup Analysis And Sensitivity Analysis}


The network meta-regression will be conducted to explore the sources of heterogeneity by using random effects models. We will also perform subgroup analyses based on the following items.

1. Age of participants: $<18,18$ to $40,50,60,>60$ years

2. Interface (helmet compared to oronosal or full-face mask) or tracheotomy or tracheal intubation

3. Degree of hypoxia

4. Study design type

5. Comorbidities (diabetes, obesity, chronic kidney disease, congestive heart failure, chronic obstructive pulmonary disease, asthma, chronic liver disease, hypertension, depression, actively treated malignancy, immunosuppression (due to illness or meds))

6. Type of healthcare setting (secondary or tertiary)

7. NIV or invasive ventilation

For all outcomes, we will first include all the trials regardless of peer reviewed or non-peer reviewed, and then we will conduct sensitivity analysis limited to peer reviewed researches. Sensitivity analysis will be conducted to explore the source of heterogeneity and ensure that a stable and accuracy conclusion can be obtained.

\section{Patients and public involvement}

All data were extracted from the literature and there will be no patients and public will be directly included in this systemic review and network meta-analysis.

\section{Discussion}

COVID-19 has spread around the world and become a global public health security problem. With limited treatment available, a large number of critically ill patients need ventilator support treatment, and the demand for ventilators has increased sharply. Although some studies have advocated early tracheotomy as a way to reduce the need for ventilators, more conservative and safe ventilate strategies may be chosen as opposed to conventional treatment, given the potential for increased viral load in the air, increased risk of cross-infection, viral transmission, and infection by medical personnel.

Compared with traditional meta-analysis, NMA can simultaneously integrate and compare the results of direct and indirect comparison of multiple interventions, and can help evaluate the efficacy and safety of various ventilation modes in patients with COVID-19. NMA analysis framework consists of Bayesian method and frequency method, the Bayesian method based on the observation data of transcendental frequency distribution and the combined effect of the distribution (likelihood), the combination of the a posteriori probability distribution to obtain merger effect. Compared with the frequency method, the Bayesian framework can form the frame of decision-making support decision, to overcome the defect of the frequency method in parameter estimation.[10] Therefore, in our review, the synthesis and analysis of 
valid data will be carried out under the Bayesian framework. For each individual study, we will rigorously analyze inclusion criteria and quality scores for each study based on GRADE assessment results.

To our knowledge, this study will be the first systematic review and NMA to analyze the efficacy and safety of different ventilation modes in patients with COVID-19. Based on the available evidence, this NMA is expected to provide a ranking of the efficacy and safety of different ventilation patterns in the treatment of patients infected with COVID-19. In addition, the results of this NMA study will likely assist patients, clinicians, and researchers in selecting the most appropriate ventilation patterns and modes for patients with COVID-19 infection. Finally, it is our sincere hope that the results of this NMA will provide the best possible ventilation model for decision making in response to COVID-19.

\section{List Of Abbreviations}

COVID-19: Coronavirus Disease 2019

NMA: Network Meta-Analysis

SMD: Standard Mean Difference

CENTRAL: Cochrane Central Register of Controlled Trials

CNKI: Chinese National Knowledge Infrastructure

CBM: Biomedical Literature Database

WHO: World Health Organization

CDC: Centers for Disease Control

GRADE: The Grading of Recommendations Assessment, Development and Evaluation System

Cls: Confidence Intervals

WMD: Weighted Mean Difference

RR: Risk Ratio

NIV: Non-Invasive Ventilation

\section{Declarations}

\section{Ethics approval and consent to participate}

Ethical approval is not required due it is a literature-based study. 


\section{Consent for publication}

Not applicable.

\section{Availability of data and materials}

The datasets generated and analyzed during the current study are available from the corresponding author on reasonable request.

\section{Competing interests}

The authors declare that they have no competing interests.

\section{Funding}

This study did not receive any specific grant from funding agencies.

\section{Authors' contributions}

$\mathrm{YHZ}$ and FW have contributed equally to this review protocol. Any disagreements were resolved by consulting TZ and he revised the manuscript critically. All authors read and approved the final manuscript.

\section{Acknowledgements}

The authors thank the Chinese Evidence-based Medicine Center West China Hospital, Sichuan University for providing statistics consultation.

\section{References}

1. John Hopkins University.Coronavirus Resource Center. 2020.https://coronavirus.jhu.edu/map.html.

2. Cytel.Global coronavirus COVID-19 clinical trial tracker. 2020.https://www.covid19-trials.org/

3. McGrath BA, Brenner MJ, Warrillow SJ: Tracheostomy for COVID-19: business as usual? British journal of anaesthesia 2020.

4. Mandal A, Nandi S, Chhebbi M et al: A Systematic Review on Tracheostomy in COVID-19 Patients: Current Guidelines and Safety Measures. Indian Journal of Otolaryngology and Head \& Neck Surgery 2020:1-5.

5. Riestra-Ayora J, Yanes-Diaz J, Penuelas 0 et al: Safety and Prognosis in Percutaneous vs Surgical Tracheostomy in 27 Patients With COVID-19. Otolaryngology-Head and Neck Surgery 2020, 163(3):462-464.

6. Martin-Villares C, Perez Molina-Ramirez C, Bartolome-Benito M et al: Outcome of 1890 tracheostomies for critical COVID-19 patients: a national cohort study in Spain. European Archives of Oto-Rhino-Laryngology 2020:1-8. 
7. Young D, Harrison DA, Cuthbertson BH et al: Effect of Early vs Late Tracheostomy Placement on Survival in Patients Receiving Mechanical Ventilation The TracMan Randomized Trial. Jama-Journal of the American Medical Association 2013, 309(20):2121-2129.

8. Moher $D$, Shamseer $L$, Clarke $M$ et al: Preferred reporting items for systematic review and metaanalysis protocols (PRISMA-P) 2015 statement. Systematic Reviews 2015, 4(1):1.

9. Guyatt GH, Oxman AD, Schuenemann HJ et al: GRADE guidelines: A new series of articles in the Journal of Clinical Epidemiology. Journal of Clinical Epidemiology 2011, 64(4):380-382.

10. Nikolakopoulou A, Mavridis D, Furukawa TA et al: Living network meta-analysis compared with pairwise meta-analysis in comparative effectiveness research: empirical study. Bmj-British Medical Journal 2018, 360(k585):1.

\section{Figures}





Figure 1

PRISMA flow diagram of the study selection process. PRISMA, Preferred Reporting Items for Systematic Reviews and Meta-Analyses. 

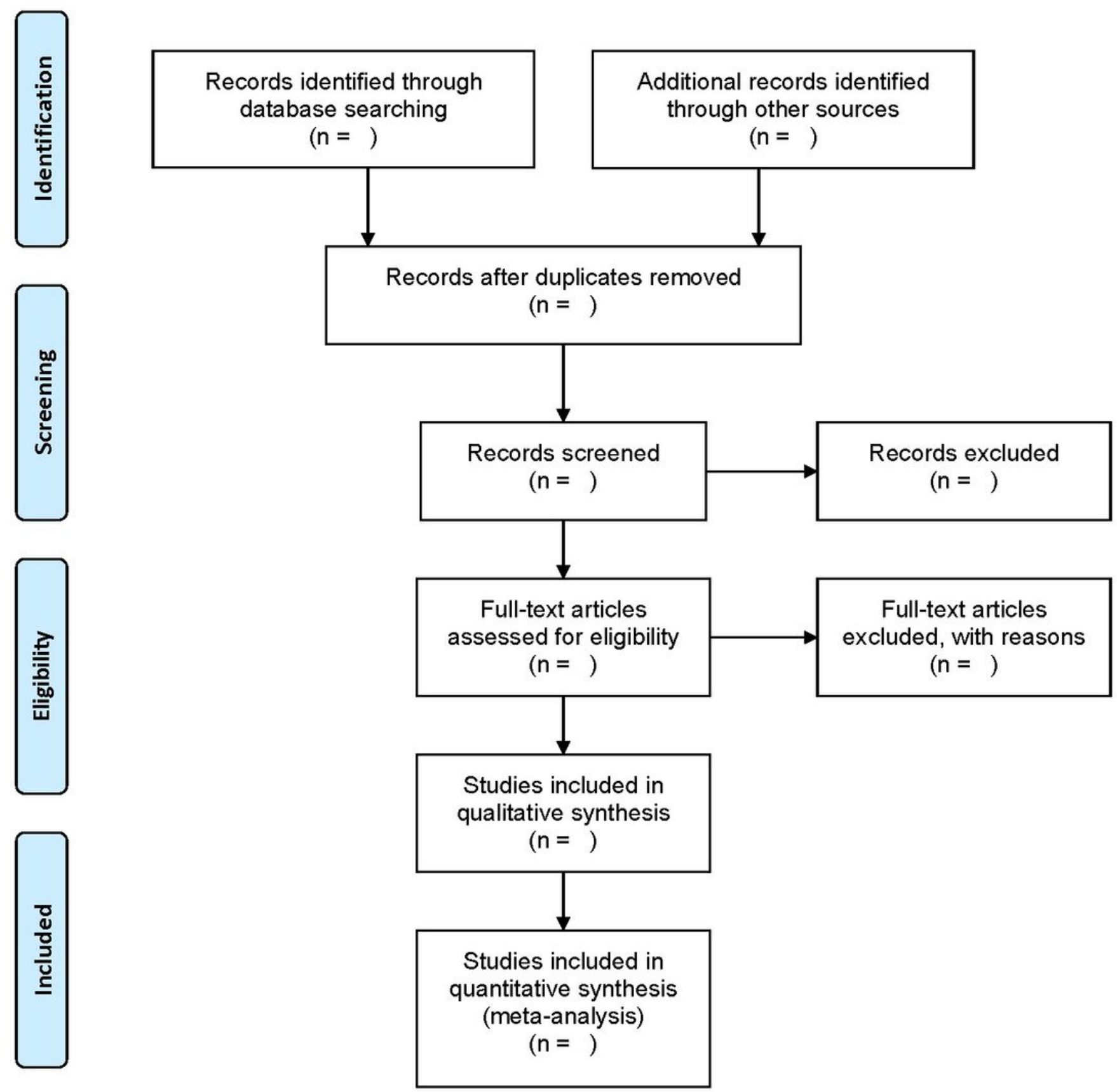

Figure 1

PRISMA flow diagram of the study selection process. PRISMA, Preferred Reporting Items for Systematic Reviews and Meta-Analyses.

\section{Supplementary Files}

This is a list of supplementary files associated with this preprint. Click to download. 
- supplymentS1.doc

- supplymentS1.doc

- supplementS2.doc

- supplementS2.doc 